\title{
Corela
}

Cognition, représentation, langage

HS-8 | 2010

L'interpellation

\section{Stratégies discursives de non-interpellation et échecs de l'interpellation dans des dialogues latins}

\section{Anna Orlandini et Paolo Poccetti}

\section{CpenEdition}

\section{Journals}

Édition électronique

URL : http://journals.openedition.org/corela/1045

DOI : $10.4000 /$ corela. 1045

ISSN : $1638-573 \mathrm{X}$

\section{Éditeur}

Cercle linguistique du Centre et de I'Ouest - CerLICO

Référence électronique

Anna Orlandini et Paolo Poccetti, « Stratégies discursives de non-interpellation et échecs de l'interpellation dans des dialogues latins », Corela [En ligne], HS-8 | 2010, mis en ligne le 22 octobre 2010, consulté le 20 avril 2019. URL : http://journals.openedition.org/corela/1045 ; DOI : 10.4000/ corela.1045

Ce document a été généré automatiquement le 20 avril 2019

\section{(i) (2)}

Corela - cognition, représentation, langage est mis à disposition selon les termes de la licence Creative Commons Attribution - Pas d'Utilisation Commerciale - Partage dans les Mêmes Conditions 4.0 International. 


\title{
Stratégies discursives de non- interpellation et échecs de l'interpellation dans des dialogues latins
}

\author{
Anna Orlandini et Paolo Poccetti
}

\section{L'acte illocutoire d'interpellation vs. non-interpellation}

1 Qu'il nous soit permit un très bref rappel étymologique pour expliquer les mots “interpellation" et "interpeller" qui n'ont pas d'équivalents dans d'autres langues romanes (pas en italien, en tout cas, qui ne connaît que "interpellanza" ("interpellanza parlamentare"), "interpellare" au sens juridique). En latin, interpellare signifie essentiellement "interrompre", et en particulier, dans un dialogue, "couper la parole

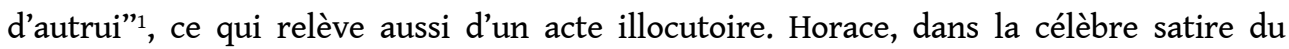
fâcheux, ne pouvant plus tolérer les vantardises de celui-ci, se dit que finalement: interpellandi locus hic erat (sat. 1,9,26) “c'était bien le moment de l'interrompre!". Toutefois, le sens du mot français est plutôt celui du latin appellare, "adresser la parole à quelqu'un, surtout pour le saluer" et de ce verbe nous produirons des témoignages chez les Comiques. On peut supposer que, par effet d'un barbarisme, on a commencé à employer interpellare à la place de appellare et de là le mot serait passé en français ${ }^{2}$. De son

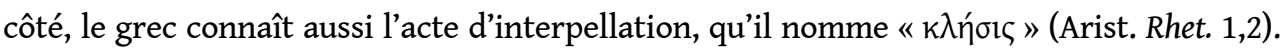
L'interpellation concentre en elle-même toutes les difficultés d'une séquence d'ouverture —qui est déjà "un moment de haute tension"3 - agrandies parce qu'il s'agit d'un acte explicite, direct, non ambigu, avec toute l'écrasante responsabilité des premiers mots. Elle sert à “ 'donner le ton', opérer une première mais décisive 'définition de la situation d'énonciation " ${ }^{4}$. Dans ce sens, la façon d'interpeller est significative du rapport que le locuteur construit. 
2 Ce n'est pas un hasard si Énée, qui n'ose pas interpeller Didon pour lui dévoiler qu'il va la quitter, se demande angoissé :

(1)Heu quid agat? Quo nunc reginam ambire furentem /audeat affatu? quae prima exordiasumat? (Verg. Aen. 4, $283 \mathrm{~s}$ )

"Hélas, que faire? Par quels discours oserait-il entreprendre une reine passionnée?

Quels mots trouver pour commencer?"

3 Si l'interpellation est un acte illocutoire (l'acte de s'adresser directement à quelqu'un pour solliciter l'intersubjectivité comme condition nécessaire et préalable à l'échange dialogal, en dégageant ainsi l'attente d'une "coopération énonciative" ${ }^{5}$ de la part de l'interlocuteur), la non-interpellation (le choix de ne pas s'adresser directement à quelqu'un) est aussi un acte illocutoire et un acte marqué. Nous montrerons que la "noninterpellation" est un phénomène de "macro-pragmatique", qui réalise une fonction discursive concernant le locuteur et l'interlocuteur, elle répond à un code, et elle est régie par des règles conversationnelles non aléatoires.

4 Le locuteur ainsi que l'interlocuteur peuvent varier en fonction de la situation d'énonciation. L'interlocuteur n'existe qu'en ce qu'il est désigné comme tel par le locuteur. Pour qu'il existe, il faut que le locuteur lui confie ce statut à plein titre. La noninterpellation est précisément l'acte de refuser d'attribuer ce statut plein; cet acte engendre une variation concernant le statut de l'interlocuteur qui est réduit à simple "destinataire", quasiment sans droit de parole, en tout cas sans droit de "coopération énonciative". C'est un acte illocutoire volontaire, pris en charge par le locuteur, qui choisit de ne pas s'adresser directement à son interlocuteur, sans pourtant oublier qu'il est présent dans la situation d'énonciation et qu'il veut lui transmettre un message. C'est aussi un acte complexe, dont la force illocutoire est de nature dérivée : le locuteur réalise des assertions qui ne sont pas purement descriptives, véri-conditionnelles: elles sont énoncées dans un but argumentatif, pour exprimer une injonction indirecte, une menace, une flatterie,, une invitation, etc. (par exemple, l'exclamation "on ne respire pas dans cette salle!" = "ouvrez la fenêtre !"). Si le "tu” est le pronom "présupposé" par le locuteur pour interpeller, la non-interpellation, qui vise à neutraliser ce "tu", se configure comme un acte illocutoire "d'indirection", qui laisse non saturées certaines composantes essentielles au dialogue. Il s'agit du cas, assez rare d'ailleurs, où l'interlocuteur et le destinataire doivent être distingués quant à leurs rôles pragmatiques même s'ils les concentrent dans un seul et unique individu. présent dans le face-à-face.

5 La non-interpellation se présente parfois comme une échappatoire temporaire et indirecte afin d'écarter les difficultés d'une approche jugée comme trop brutale ou en tout cas trop directe. D'ailleurs, l'indirection est à la source de toutes les stratégies d'interaction visant à réduire les aspects menaçants pour la face positive de l'interlocuteur, mais aussi pour la face positive du locuteur ${ }^{6}$.

Dans le langage conversationnel de la comédie grecque et latine, les personnages ont recours à des moyens différents pour s'adresser à l'interlocuteur; chacun, selon sa couche d'appartenance, peut choisir la forme d'interpellation qui s'adapte mieux à son niveau culturel et social ${ }^{7}$. D'une manière analogue, les personnages sur scène peuvent exploiter des stratégies différentes de non-interpellation ou de rejet de l'interpellation qui correspondent à leur niveau social ; ainsi, par exemple, comme nous le verrons dans la suite, pour rejeter l'interpellation qui vient d'un esclave on peut répondre par un simple silence, dénonçant de cette manière un statut social et un rôle de pouvoir 
supérieur. La relation verticale de pouvoir (le pouvoir d'interpeller) est ainsi remise en cause, voire rejetée.

7 Les conditions socio-pragmatiques qui poussent à éviter ou à rejeter l'interpellation peuvent être disparates et parfois opposées entre elles : rôle socialdifférent, difficulté à tutoyer, politesse, émotivité aiguë dans un contexte conflictuel, etc.

8 Ce dernier cas est très fréquent et les stratégies discursives que l'on exploite semblent être universelles. Pour ne pas pousser trop loin notre agressivité, ou pour ne pas perdre trop manifestement la face, par une "loi de prudence", mais aussi par une "loi de dignité" 8, l'on efface de la communication l'autre en tant qu'interlocuteur, l'on fait comme s'il était transparent. De la deuxième personne, il est abaissé au rang de la troisième (la nonpersonne d'É. Benveniste, 1966), sans droit de réplique. On lui parle et on ne lui parle pas vraiment.

9 On évite ainsi toute modalité directe : les injonctifs, les impératifs, les vœux, les requêtes, aussi bien que les questions. On ne fait que des assertions et elles aussi le plus possible génériques, mais dotées d'une force illocutoire indirecte.

10 Comme au téléphone, lorsque quelqu'un s'annonce ex abrupto : "Je cherche mon fils", pour court-circuiter la personne qui effectivement répond à l'appel, comme si elle n'existait pas, en effaçant d'un coup tous les rituels que la politesse demande pour l'ouverture d'une communication. Toutefois, celui qui répond demeure la cible de l'acte complexe et le destinataire de l'injonction indirecte "Passez-le moi!" et il peut toujours enchaîner sur le même ton, sans salutations préalables, sans rituels de politesse, par une autre assertion: "Il n'est pas là" ou "Je vais lui dire". Il existe, en effet, une graduation, une scalarité, dans les règles de politesse lorsqu'il s'agit de s'adresser en ouverture de l'échange dialogal. Cette scalarité peut être ainsi hiérarchisée : si appellatif de la personne à laquelle l'on s'adresse et verbe à l'impératif représentent la formule standard; l'impératif seul témoigne d'un degré de politesse inférieur et il peut être aperçu comme hostile ou impoli'; enfin, l'emploi de la troisième personne est la marque de la noninterpellation.

11 Lewis Carroll, vrai génie des règles conversationnelles, montre bien l'importance des premiers mots dans la rencontre d'Alice et du Chapelier ${ }^{10}$ :

(2a) "Il faut vous faire couper les cheveux, dit le Chapelier. Il fixait Alice depuis quelque temps avec une intense curiosité et c'étaient là ses premières paroles.-On ne doit pas faire des remarques personnelles, dit Alice sévèrement, c'est très impoli" et ce l'est d'autant plus qu'elles figurent dans les "premières paroles" (Alice au pays des merveilles, Marabout 1963, 86-7).

12 Le Chapelier interpelle d'emblée, directement la petite fille en la vouvoyant et en lui adressant des injonctions de caractère personnel; cela est reçu comme un acte très impoli par Alice qui rejette ce genre d'interpellation qui lui est adressée et l'évite à son tour, ne permettant pas la réalisation d'un véritable échange dialogal, et se bornant à faire des assertions génériques à la troisième personne, que, toutefois, le Chapelier devra comprendre comme un reproche d'impolitesse.

13 À la place du pronom personnel de la deuxième personne - en latin surtout Tu en position incipitaire :

(2b) Tu ne quaesieris ... Leuconoe (Hor. carm. 1, 11, 1-2)

"Ne recherche point toi, ... Leuconoé"

(2c) $\mathrm{Tu}$ a ciuitatibus pecunias classis nomine coegisti, $\mathbf{t u}$ pretio remiges dimisisti, tu

archipiratam ab oculis omnium remouisti, tu ...(Cic. Verr. II 5,136) 
“C'est toi qui, sous prétexte d'équiper la flotte as perçu des sommes d'argent des cités; c'est toi qui à prix d'or as envoyé en congé les matelots; c'est toi qui, un navire de corsaire ayant été capturé par ton questeur et ton lieutenant, as soustrait à la vue de tous le chef des pirates, c'est toi qui ..."

(2d) - Tu in forum prodire, tu lucem conspicere, tu in horum conspectum uenire conaris? ( Rhet. Her. 4,13,19) "Tu veux paraître au forum? Tu veux voir la lumière ? Tu veux te présenter à la vue de ces hommes?" est aussi très fréquent: tel est le cas, par exemple, du dialogue entre Philocomasie et l'esclave Scélédrus, dans le Miles Gloriosus de Plaute; Philocomasie est furieuse contre Scélédrus qui l'a surprise dans la maison du voisin en train d'embrasser un jeune homme :

(3a) PH. Tun me uidisti? SC. Atque his quidem hercle oculis ...PH. Carebis, credo, / qui plus uident quam quod uident. / SC. Numquam hercle deterrebor quin uiderim id quod uiderim. PH. Ego stulta et mora multum, quae cum hoc insano fabuler, quem pol ego capitis perdam (Plaut. Mil. 368-371)

“Tu m'as vue?":: “Oui, et de mes yeux":: “Tu les perdras, je crois bien : ils voient plus qu'il n'y a à voir"::”Jamais, par Hercule, on ne m'empêcheras d'avoir vu ce que j'ai vu":: “Je suis trop sotte et folle de parler avec ce dément, dont j'aurai la tête, par Pollux!"

On voit ici, exploité par Philocomasie, le "trope communicationnel" qui sera une constante de tout acte de non-interpellation et qui "consiste à feindre de ne pas affronter directement celui qu'on attaque verbalement: pour éviter qu'il s'en prenne "plein la gueule", on parle "dans son dos"11. Scélédrus toutefois, entend très bien la menace qui lui est adressée d'une manière indirecte, et il répond continuant de tutoyer Philocomasie:

(3b) SC. Noli minitari (Plaut. Mil. 372)

"Ne me menace pas"

\subsection{Dans un contexte de détente : l'interpellation évitée par politesse}

Mais l'on choisit aussi la non-interpellation lorsqu'on veut rétablir des conditions de bonne entente, lorsqu'on veut faire la paix, après une situation conflictuelle, on prend alors mille précautions envers la face positive de l'autre. On pourrait citer un poème d'Horace, $(3,9)$ qui célèbre la réconciliation des amants, notamment Horace et Lydie. Au début du dialogue, ils s'étaient réciproquement offensés en se déclarants très heureux et comblés par leurs partenaires actuels : Chloe et Calais. Surtout Lydie avait renchéri en se disant prête "à mourir deux fois, si les dieux sauvent son beau garçon" pro quo bis patiar 
mori, / si parcent puero fata superstiti (v. 15 et 16). À ce point, la situation est très tendue, la dispute pourrait mal tourner, même si, comme le dit Térence: Amantium irae amoris integratio est "la colère des amants est un enrichissement de l'amour" (Andr. 555). Pour apaiser Lydie, au lieu de l'interpeller directement pour lui proposer de se remettre ensemble, Horace prend mille précautions visant à sauvegarder la face de l'amie, et la sienne aussi, en cas de réponse négative :

(4a) "Quid si prisca redit Venus/ diductosque iugo cogit aeneo?/ Si flava excutitur

Chloe/ reiectaeque patet /ianua Lydiae ?" (Hor. carm. 3,9,17-20)

"Et si l'amour d'autrefois revenait ... et si la blonde Cloé était congédiée ....?"

Remarquons que le prédicat excutitur est un passif sans l'agent exprimé (Cloéest "laissée tomber" d'elle-même), et la porte aussi semble s'ouvrir toute seule (ianua patet), comme si Horace s'était effacé lui-aussi, non seulement son interlocutrice. Mais elle a bien reçu le message indirect et le but est atteint ; c'est le moment de revenir au "tu" :

(4b) Tecum uinere amem, tecum obeam libenter (Hor. carm.3,9,24)

"C'est avec toi que je voudrais vivre, c'est avec toi que je mourrais volontiers"

Inversement, le choix du mode impersonnel et le passage de la deuxième à la troisième personne peut aussi se réaliser, tout au milieu du dialogue, dans une situation conflictuelle ; c'est le cas qu'on rencontre dans une tragédie de Sénèque, Médée ${ }^{12}$ :

(5) Quoi me remittis ? exuli exilium imperas, / nec das. 'Eatur'. Regius iussit gener. (Sen. Med: 459-460)

"Où me renvoies-tu? Tu m'imposes l'exil ; mais tu ne m'en indiques pas le lieu. “

Qu'elle s'en aille": voilà ce qu'ordonne le gendre de Créon".

$21 \mathrm{Au}$ milieu de son discours à Jason, Médée opère un double changement dans l'énonciation; elle imagine une parole de celui-ci, qu'elle nomme, dans sa colère, à la troisième personne pour l'éloigner d'elle (non-interpellation de Médée à l'égard de Jason); mais elle-même dans le discours supposé de Jason n'est nommée qu'indirectement (non-interpellation de Jason à l'égard de Médée). Dans un cas, l'impersonnel "Eatur" souligne l'attitude rejetante dans la bouche de Jason par rapport à Médée. À son tour, Médée répond avec le même procédé d'éloignement à l'égard de Jason (regius iussit gener), comme s'il était devenu une troisième personne, un autre.

\subsection{L'interpellation retardée}

Souvent, pour faire augmenter la tension ainsi que le ton menaçant, l'on évite, dans un premier temps, l'interpellation directe. Tout en faisant semblant de ne pas voir l'interlocuteur, l'autre lui fait parvenir des menaces indirectes, mais absolument claires. C'est à nouveau l'exploitation du "trope communicationnel". Un acte préparatoire d'un autre acte illocutoire.

Un exemple célèbre est la rencontre de Mercure et de Sosie devant la maison d'Amphitryon. Il s'agit d'“à parté" où l'interpellation se fait via le public, mais elle s'adresse aussi à l'interlocuteur (qui n'est pas encore tel) et elle est néanmoins chargée de menaces qui attendront leur cible.

Ainsi d'un premier abord, Mercure n'interpelle pas directement Sosie, il lui parle par sententiae adressées à ses poings, d'une voix forte, à la cantonade:

(6a) ME Agite, pugni, iam diu est quod uentri uictum non datis Plaut Amph..302)

“Allons, mes poings: il y a longtemps que vous n'avez donné de quoi vivre à mon ventre"...

(6b) ME Quisquis homo huc profecto uenerit, pugnos edet Plaut. Amph...309) 
"Quiconque viendra ici mangera mes poings" ...

(6c) ME Haud malum huic est pondus pugno Plaut. Amph..312)

"Mon poing pèse un assez bon poids"

(6d) ME gestiunt pugni mihi Plaut. Amph..323)

"Les poings me démangent"

\section{dit et reçu :}

(8) ME Quo ambulas tu, qui Volcanum in cornu conclusum geris? SO. Quid id exquiris tu, qui pugnis os exossas hominibus? (Plaut. Amph.. 341-342)

“Où vas-tu, toi, qui porte Vulcain enfermé dans une corne?":: “Que viens -tu chercher, toi, qui de tes poings désosses le visage des gens?"

Toutefois, la même stratégie peut aussi bien être exploitée dans un contexte qui n'est pas du tout ménaçant, bien au contraire, elle peut être utilisée plutôt dans le but de séduire. Ainsi dans Miles Gloriosus de Plaute, lorsqu'Acrotéleutie feint d'être amoureuse du Soldat, elle parle à haute voix, pour se faire entendre du destinataire du message, présent sur scène, mais sans l'interpeller directement :

(9a) AC. Si pol me nolet ducere uxorem, genua amplectar / atque obsecrabo. Alio modo, si non quibo impetrare, / consciscam letum; uiuere sine illo me non posse (Plaut. Mil. 1239-1241)

“Si, par Pollux, il refuse de m'épouser, j'embrasserai ses genoux, et je le supplierai. Autrement, si je ne puis obtenir ce que je souhaite, je mettrai fin à mes jours ; je sais que je ne puis vivre sans lui".

Acrotéleutie est bien rusée, elle avait même pris la précaution d'inviter son interlocuteur, Palestrion, à parler à haute voix pour que le Soldat, qu'elle refuse encore d'interpeller, entende :

(9b) AC. Ne parce nocem, ut audiat! (Plaut. Mil.1220) “Ne ménage pas ta voix , qu'il entende"

et elle va:encore plus loin, prétendant qu'elle sent par son nez la présence du bien aimé ("Je sens une odeur ...mon nez me dit...") :

(9b) AC . non est intus quem ego uolo. MI.Qui scis? AC Scio (de olefacto) / nam odore nasum sentiat, si intus sit (Plaut. Mil.1255 s.)

31 “... l'homme que je veux n'est pas dans la maison." :: “Comment le sais-tu?” :: “Je le sens, je le sais ; mon nez en percevrait l'odeur, s'il était dans la maison"

32 Drôle de dialogue à distance, par un intermédiaire, sans interpellation directe, donttoutefois s'accommode aussi le Soldat :

(9c) PA. Vt, quaeso, amore perditast te misera! PY. Mutuum fit. / PA: Tace, ne audiat. sit (Plaut. Mil.1253 s.) "Comme elle est éperdue d'amour pour toi, s'il te plaît, la pauvre!” :: “Je le lui rends bien” : “Tais-toi, qu'elle n'entende pas!” 
Drole de réponse de la part de Palestrion, feignant la précaution, par souci qu'Acrotéleutie n'entende pas : et, pourtant, tous les trois, sont bien tous ensemble sur scène.

Un autre exemple de cette stratégie nous est fourni par la tragédie. Chez Sénèque, Phèdre, au moment précis où elle va avouer que c'est bien Hippolyte -son interlocuteurqui est l'objet de son amour, s'adresse à sa sœur Ariane et c'est le dernier mot (gnatus) qui révèle le vrai destinataire de l'énonciation ainsi que de sa passion, jusqu'alors mentionné à la 3ème personne: l'aveu explicite, repoussé jusque-là, se fait sous cette forme légèrement indirecte :

(9d) in te (i. e. Hipp) magis refulget incomptus decor:/ est genitor in te totus et toruae tamen pars aliqua matris miscet ex aequo decus:/ in ore Graio Scythicus apparet rigor / si cum parente Creticum intrasses fretum,/tibi fila potius nostra neuisset soror./Te te, soror (i. e. Ariane), quacumque siderei poli/in parte fulges, inuoco ad causam parem:/domus sorores una corripuit duas / te genitor, at me gnatus. en supplex iacet/ adlapsa genibus regiae proles domus./respersa nulla labe et intacta, innocens/tibi mutor uni.certa descendi ad preces:/ finem hic dolori faciet aut uitae dies./miserere amantis. (Sen. Phaed.657-671)

"Ce costume plus simple relève encore votre beauté. À tout ce qui charmait dans votre père, vous joignez les grâces un peu sauvages de votre mère; c'est la beauté du jeune Grec relevée par la fierté un peu farouche d'une Amazone. Ah! si vouseussiez suivi votre père sur les mers de la Crète, c'est à vous que ma sœur eût remis le fil sauveur. ô ma sœur, en quelque partie du ciel que tu brilles, favorise une ardeur semblable à la tienne. Nous avons trouvé notre vainqueur dans la même famille. Le fils m'inspire l'amour que tu ressentis pour le père. À ses pieds la fille d'un roi puissant. Jusqu'aujourd'hui innocente et pure, c'est pour vous seul que je trahis mes devoirs. C'en est fait, ma résolution est prise, vous avez entendu ma prière. Ce jour terminera ou ma peine ou ma vie. Oh! prenez pitié d'une infortunée qui vous aime."

Phèdre parle d'elle-même à la 3ème personne aux v. 666-67 (regiae proles), avant de retrouver le dialogue direct in fine (tibi / miserere). En ce cas, il s'agit d'une forme d'atténuation, comme pour l'agressivité mais ici pour l'amour, pour retarder et adoucir le choc de l'aveu direct à son beau fils (tibi mutor uni) ! Et Hippolyte de lui répondre.

\section{De la part de l'interlocuteur : les échecs de l'interpellation}

\subsection{L'interpellation rejetée en lui opposant le silence}

Parmi les échecs de l'interpellation, on compte aussi le cas où l'interlocuteur n'accepte pas l'échange, il ne reconnaît pas à l'autre un statut de pouvoir qui lui confère le droit de l'interpeller.

Dans le Miles de Plaute, Philocomasie feint, à un moment donné, de ne pas être elle-même, mais sa sœur jumelle venue d'Athènes, et de ne pas reconnaître Scélédrus qui l'interpelle, alors qu'elle lui oppose d'abord un silence énigmatique :

(9a) SC. Adeamus, appellemus. Heus, quid istuc est, Philocomasium?/ Quid tibi istic in <ist>isce aedibus debetur? quid negotist? / Quid nunc taces? tecum loquor. PA: Immo edepol tute tecum ; / nam haec nihil respondet.(Plaut. Mil.420-423)

SC. “Abordons-la, adressons-lui la parole.Hé, qu'est cela, Philocomasie? Qu'est -ce

Corela, HS-8 | 2010 
que tu as à réclamer dans cette maison-ci? Quelle affaire y as-tu? Pourquoi ne dis-tu rien? Je te parle!" :: "Tu te parles plutôt à toi-même, par Pollux ; car elle ne répond mot" et il ne dure pas longtemps. C'est le cas aussi de notre pièce, où la femme qui se feint une étrangère finit par interrompre son silence outré, tout en niant à l'autre le droit d'être interpellée comme si elle était Philocomasie:

(11) SC. Te adloquor, uiti probrique plena, /, quae circum uicinos uagas. PH. Quicum tu fabulare? / SC. Quicum nisi tecum? / Quis tu homo es? aut mecum quid est negoti? / SC. Me 
rogas, hem, qui sim? (Plaut. Mil.420-426)

"C'est à toi que je m'adresse, femme éhontée, vicieuse, qui vas faire le tour des voisins" :: “À qui parles-tu, et qu'ai-je à faire avec toi?":: “Tu me demandes qui je suis, hein?"

\subsection{L'interpellation rejetée parce que jugée comme inadéquate par rapport à la situation discursive}

\section{réalité est Jupiter déguisé) , refuse cette interpellation comme non adéquate à la situation} discursive :

(12b) AL. Obsecro ecastor, quid tu me deridiculi gratia / sic salutas atque appellas, quasi dudum non uideris, / quasi qui nunc primum recipias te domum huc ex hostibus ? Plaut. Amph.682-684

“Ah, je t'en prie, pourquoi te moques-tu de moi en me saluant et en m'interpellant ainsi, comme si tu ne m'avais pas vue depuis longtemps, comme si c'était maintenant la première fois que tu rentrais à la maison après la guerre ?"

\section{4. Échecs de l'interpellation par ambiguïté}

Parfois, lorsqu'il s'agit de noms propres qui ont un sens non arbitraire (c'est-à-dire qu'ils prédiquent des propriétés de l'individu), l'interpellation peut être mise en échec en jouant sur l'ambiguïté entre la valeur référentielle du nom propre, en tant que désignateur rigide ("Le seul individu appelé X ") et la valeur prédicative de la qualité à laquelle le nom renvoie par son propre sémantisme. Il s'agit d'une ambiguïté qui se répercute sur la force illocutoire différente d'un acte appellatif et d'une connotation appositive. C'est le cas, par exemple du mot Truculentus qui sert aussi bien à nommer un individu (" (Le) Brutal ") ou à prédiquer un vice (" être brutal ") :

(13a) AST. Nimis quidem hic truculentust TR. Pergin male loqui, mulier, mihi ? AT. Quid tibi ego maledico ? TR. Quia enim me truculentum nominas [...] (Plaut. Truc. 265 ss.).

“Tu es vraiment trop brutal " : : "Tu continues à m'insulter, femme ?" : : En quoi est-ce que je t'insulte ?" : : En m'appellant Brutal ...."

L'emploi du verbenominare dans la formule figée me X nominas oriente vers l'acte d'appellation, mais qualifier quelqu'un de Truculentus peut aussi dire de quelqu'un qu'il a le vice d'être brutal. Dans ce passage l'ambiguïté est volontaire. Et ainsi Truculentus de poursuivre son jeu, exploitant à nouveau le jeu entre la qualification et l'appellation, qui renvoie à son identité personnelle:

(13b) TR. nimio minus saevos iam sum, Astaphium, quam fui. Iam non sum truculentus, noli metuere (Plaut. Truc. 674)

“Je suis bien moins terrible que je n'étais autrefois, Astaphie. Je ne suis plus Brutal, n'aie pas peur"

Dans un autre cas, une ambiguïté, cette fois-ci involontaire, concerne un nom propre (pour mieux dire un ethnique Cauneas, "figues de Caunos", employé comme nom 
commun : "des figues") qui pourrait coïncider, pour ceux qui savent entendre, avec une phrase entière: une interpellation adressée à Crassus sur le point de partir pour la guerre, l'invitant à éviter de le faire:

(14) Cum M. Crassus exercitum Brundisi imponeret, quidam in portu caricas Cauno aduectas uendens "Cauneas ${ }^{16 ! " ~ c l a m i t a b a t . ~ D i c a m u s, ~ s i ~ p l a c e t, ~ m o n i t u m ~ a b ~ e o ~ C r a s s u m ~}$ caueret ne iret ; non fuisse periturum, si omini paruisset (Cic. diu. 2,84)

“Alors que M.Crassus imbarquait son armée à Brindes, un vendeur de fruits criait "Cauneas". Disons, si Crassus avait compris cette expression comme :

" prends garde de ne pas y aller!" il aurait eu la vie sauvée".

Ce passage nous révèle que, dans un registre peu soigné de prononciation, l'expression caue ne eas "(toi), prends garde de ne pas y aller!" pouvait aboutir à Cauneas ! ${ }^{17}$ (dans le contexte du vendeur : le nom de figues provenant de la région de Caunos).

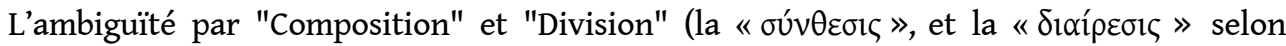
Aristote (Réfutations sophistiques (IV, 165b 24)) a mis en échec l'interpellation avec des résultats funestes.

51 Comme dernier exemple d'une ambiguïté savoureuse par le refus de l'interpellation, rappelons enfin une scène du célèbre Cyrano de Bergerac: un petit marquis vaniteux et prétentieux avait été ridiculisé par Cyrano; le marquis hors de lui lance à Cyrano une bordée d'injures, notamment « Faquin! » et Cyrano met en échec ce type d'interpellation offensive, feignant de croire que le marquis vient de décliner son identité, comme cela se faisait avant un duel, en s'écriant : "Ah ? et moi Savignien-Hercule, Cyrano de Bergerac !". Ou si vous préférez, la version de nos jours: "Connard!" - "Enchanté. Moi, c'est Jacques Chirac!", bel exemple d'esprit cultivé qui avait le sens de la repartie et faisait appel à la memoria docta pour rejeter avec élégance l'interpellation injurieuse, et sans en avoir l'air, et faire retomber l'offense sur celui qui l'avait déclenchée. Il s'agit d'une suite de deux actes linguistiques ayant une force illocutoire différente : au premier, qui lance l'injure, on riposte par un acte illocutoire complexe, le refus de l'interpellation, qui se dédouble dans une interprétation de premier degré, pseudo-naïve, attribuant au premier acte une force présentative (la déclinaison d'identité) auquel se superpose, de manière implicite mais perçante, un nouvel acte d'injure qui joue sur le même mot.

\section{Conclusion}

Comme la plupart des actes illocutoires négatifs, l'acte de non-interpellation ou le rejet d'une interpellation sont des actes marqués et ils demandent, le plus souvent, une interprétation complexe ; en particulier pour les actes d' "indirection", qui peuvent être orientés vers un but aussi bien positif que négatif; ce que nous avons essayé de montrer en nous appuyant sur des nombreux contextes dialogaux issus des comédies latines.

\section{BIBLIOGRAPHIE}

Adams, John N. (1978). Conventions of Naming in Cicero. Classical Quarterly NS 28, pp. 145-166. 
Bilmes, Jack, (1994). Constituting Silence. Life in a world of total meaning, Semiotica 98, pp. 73-87. Braun, F. (1988). Terms of Address : Problems of Patterns and Usage in Various Languages and Cultures, Berlin.

Brenneis, Donald, (1988). Language and Disputing, Annual Review of Anthropology 17, 221-237.

Cortini, Michela, (2001). Silence as a tool for negotiation of sense in multi-party conversation, in E. Weigand - M. Dascal (eds.), Negotiation and Power in Dialogic Interaction. Current Issues in Linguistic Theory 214, pp. 167-180. Amsterdam-Philadelphia, J. Benjamins.

Détrie, Catherine (2006). De la non-personne à la personne : l'apostrophe nominale. Paris, CNRS éditions.

Dickey, Eleanor, (1995). Forms of addressand conversational language in Aristophanes and Menander, Mnemosyne XLVIII, pp. 257-271.

Dickey, Eleanor, (1996). Greek Forms of Address, Oxford : Oxford University Press.

Dickey, Eleanor, (2002, paperback 2007). Latin Forms of Address, Oxford : Oxford University Press.

Goffman, Erving, (1974). Les Rites d'interaction. Paris, Minuit.

Goffman, Erving, (1976). “Replies and responses”, Language in Society 5, pp. 257-313.

Goffman, Erving, (1981). Forms of Talk, Philadelphia, University of Pennsylvania Press.

Grice, Herbert.Paul (1975). Logic and Conversation, in P. Cole - J. L. Morgan (eds.) Syntax and Semantics. Vol.3, New-York-San Francisco-London, Academic Press, pp. 41-58.

Kerbrat-Orecchioni, Catherine, (1984). Pour une approche pragmatique du dialogue théatral. Pratiques 41, pp. 46-62.

Kerbrat-Orecchioni, Catherine, (1986). L'implicite. Paris,Armand Colin.

Kerbrat-Orecchioni, Catherine, (1990). Les interactions verbales, vol.I, Paris,Armand Colin.

Kerbrat-Orecchioni, Catherine, (1992). Les interactions verbales, vol.II, Paris,Armand Colin.

Kurzon, Denis, (1992). When Silence May mean Power, Journal of Pragmatics 18, pp. 92-95.

Lakoff, Robin, (1973). The Logic of Politeness. Papers from the ninth regional meeting of the CLS ed. by C.Corum, T.C. Smith-Stark - A. Weiser, pp. 292-305. Chicago, CLS.

Laver, J. (1981). Linguistic Routines and Politeness in Greeting and Parting, in Coulmas (éd.) Conversational Routine, La Haye-Paris-New York, Mouton, pp. 289-304.

Roulet. Eddy, (1980). Modalité et illocution, Communications 32, pp. 216-239.

Turnbull, William \& Peter Muntigl (1998). Conversational Structure and Facework in Arguing, Journal of Pragmatics 8, pp. 23-43.

\section{NOTES}

1. Selon Ps. Ascon. Verr. p. 241,22 interpellare est mediam alterius orationem interrumpere uel actioni se alterius interponere.

2. . Selon Ps. Iulian. Tol. gramm. V 324,18 barbarismus sicut ... interpella pro appella.

3. . Laver $(1981,291)$.

4. Kerbrat-Orecchioni $(1990,221)$.

5. . Cf. Détrie 2006. 
6. . En ce qui concerne les règles de politesse qui s'articulent sur la théorie des "faces" "Face threatening Act”, cf . Goffman 1974 ; Roulet 1980 ; Kerbrat-Orecchioni 1984 ;1986; 1990 et 1992.

7. . Cf. Braun 1988, 184 et Dickey 1995, 1996 et 2002.

8. . Cf. Kerbrat-Orecchioni $(1986,235)$.

9. . Cf Adams $(1978,163)$ pour le latin de Cicéron.

10. Texte cité par Kerbrat-Orecchioni $(1990,222)$.

11. . Kerbrat-Orecchioni $(1986,280)$.

12. . Ce passage, tout comme plus haut Sen. Phaed.657-671 =(9d), nous a été signalé par un collègue et ami de grande sensibilité linguistique, Jean-Pierre Aygon; qu'il soit ici vivement remercié.

13. . Cf. Kurzon (1992,92-94).

14. . Cf. Lakoff, R. 1973.

15. . Cf. Bilmes 1994, Kurzon 1992, Turnbull \& Muntigl 1998, et, en particulier, Cortini 2001.

16. . Cauneas (sc. ficus uendo / emite) ; cf. MADVIG apudPEASE $(1920-23=1963,485)$

17. L'agglutination de caue ne eas s'expliquepar la chute de $e$ dans caue et la synalèphe entre ne et eas

\section{RÉSUMÉS}

Cette enquête de macro-pragmatique vise à reconnaître les codes d'un acte illocutoire qui auparavant n'a jamais été reconnu et analysé comme tel, l'acte de "non-interpellation". Il répond pourtant à une stratégie dialogalequi préside aux interactions verbales et qui en respecte les règles. Le“trope communicationnel"est le phénomène discoursif le plus fréquent dans la noninterpellation, à côté de l'abolition des rituels de politesse qui président l'ouverture, ainsi que des signes qui attribuent à l'interlocuteur son statut plein de pouvoir interagir. Les raisons d'un échec de l'interpellation nous ont semblé aussi dignes de réflexion.

Le "silence de pouvoir", la "troncation" de l'échange, le jugement de non adéquation de l'interlocuteur, de la situation énonciative, les phénomènes d'ambiguïté, sont tous des causes de l'échec interpellatif.

This macro-pragmatic investigation aims at recognizing the codes of an illocutionary act that so far has been never identified or analyzed as such, i.e. the act of 'non-interpellation'. It corresponds to a dialogic strategy that lies behind verbal interactions and that abides by the rules of them. It appears that the choice of non-interpellation typically includes the 'communicative trope', beside the elimination of politeness procedures of the opening, as well as the signs that ascribe the interlocutor his full capacity of interaction. The reasons of such failed interpellation may be identified in the 'silence of power', in the 'truncation' of the exchange, in the judgment of non-adequacy of the interlocutor and of the speech situation, and in phenomena of ambiguity.

Quest'indagine di macro-pragmatica è volta ad individuare i codici che regolano un atto illocutorio che in precedenza non è mai stato riconosciuto come tale: l'atto di "non interpellazione". Un atto che pure corrisponde ad una determinata strategia discorsiva che presiede alle interazioni verbali e ne rispetta le regole. Abbiamo verificato che è il "tropo comunicativo" la figura più frequente nella scelta della "non interpellazione", di fianco 
all'abolizione dei rituali di educazione che presiedono all'apertura dello scambio dialogale, insieme ai segni che attribuiscono all'interlocutore il suo status di potere interattivo. Le ragioni di fallimento dell'interpellazione ci sono ugualmente sembrate degne di riflessione.

Il "silenzio di potere", la "troncazione" dello scambio dialogale, il giudizio di inadeguatezza concernente l'interlocutore, la situazione enunciativa, i fenomeni di ambiguità, sono tutte cause del fallimento interpellativo.

\section{INDEX}

Mots-clés : non interpellation, macro-pragmatique, trope communicationnel, silence de pouvoir, troncation, macro-pragmatica

Keywords : non-interpellation macro-pragmatic, communicative trope l, silence of power, truncation

\section{AUTEURS}

\section{ANNA ORLANDIN}

Université Toulouse 2 / Université Rome 2

\section{PAOLO POCCETTI}

Université Rome 2 